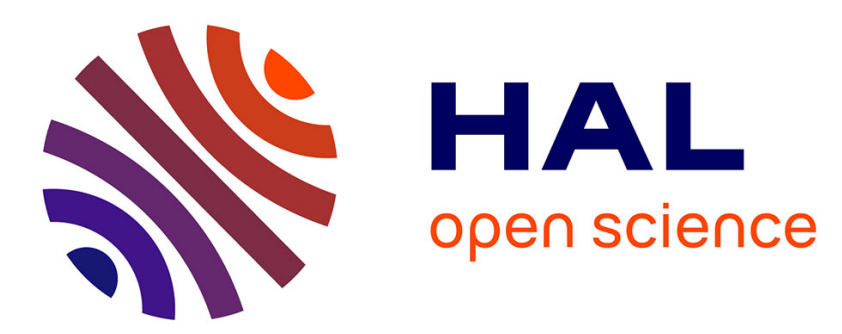

\title{
An LMI-based approach for the control of semi-active magnetorheological suspensions
}

Ruben Begnis, Giulio Panzani, Mirko Brentari, Luca Zaccarian

\section{To cite this version:}

Ruben Begnis, Giulio Panzani, Mirko Brentari, Luca Zaccarian. An LMI-based approach for the control of semi-active magnetorheological suspensions. 21st IFAC World Congress, IFAC 2020 (virtual ), Jul 2020, Berlin (virtual), Germany. hal-03027962

\section{HAL Id: hal-03027962 \\ https://hal.science/hal-03027962}

Submitted on 27 Nov 2020

HAL is a multi-disciplinary open access archive for the deposit and dissemination of scientific research documents, whether they are published or not. The documents may come from teaching and research institutions in France or abroad, or from public or private research centers.
L'archive ouverte pluridisciplinaire HAL, est destinée au dépôt et à la diffusion de documents scientifiques de niveau recherche, publiés ou non, émanant des établissements d'enseignement et de recherche français ou étrangers, des laboratoires publics ou privés. 


\title{
An LMI-based approach for the control of semi-active magnetorheological suspensions
}

\author{
Ruben Begnis * Giulio Panzani ${ }^{* *}$ Mirko Brentari ${ }^{* *}$ \\ Luca Zaccarian ${ }^{* * *, *}$ \\ * Dipartimento di Ingegneria Industriale, Università degli Studi di \\ Trento, Italy (e-mail: ruben.begnis@studenti.unitn.it). \\ ** Dipartimento di Elettronica, Informazione e Bioingegneria, \\ Politecnico di Milano, Milan 20133, Italy (e-mail: \\ giulio.panzani@polimi.it). \\ *** CNRS, LAAS, Université de Toulouse, 31400 Toulouse, France \\ (e-mail: zaccarian@laas.fr).
}

\begin{abstract}
We address modelling and control of semi-active suspensions based on magnetorheological (MR) fluids. We first introduce a saturation-based model of the underlying nonlinear phenomena, and combine the MR suspension with a quarter car model whose parameters resemble an SUV-like vehicle. Then we present simulation results to test the passenger comfort arising from the passive configuration (namely holding the input constant over the whole simulation) and the use of the Skyhook controller (which is adapted from its typical use in electrohydraulic suspensions). Finally, we propose LMI-based control designs exploiting an approximated model and typical generalized sector conditions for saturation nonlinearities. The simulation results show the advantages of the proposed design.
\end{abstract}

Keywords: Semi-active suspensions, vehicle dynamics, magnetic suspension, ride comfort, passive suspensions

\section{INTRODUCTION}

Semi-active suspensions correspond to shock absorbers whose dynamical nature can be manipulated by way of some suitable control inputs with the constraint that no energy can be injected into the system (as opposed to active suspensions that can inject energy, see Savaresi et al. (2010)). These suspensions have become popular in the automotive field because they are cost-effective solutions that can be employed to considerably increase the driving comfort.

The first developed semi-active suspensions technology consisted in the so-called linear adaptive configuration, wherein the viscous damping coefficient $c$ of an electrohydraulic $(\mathrm{EH})$ shock absorber can be adjusted in real-time between a bounded (and positive) set of allowable ranges $\left[c_{\min }, c_{\max }\right]$, by manipulating the size of the orifices within the suspension. While the passive EH configuration is fully linear (because viscous damping is a linear phenomenon), the input $c$ of the linear adaptive solution enters nonlinearly in the vehicle vertical dynamics, thereby posing interesting control design challenges for improved passenger comfort and/or road handling. Different approaches have been proposed for the control of such devices; among them, switching strategies (Savaresi and Spelta, 2007), optimal control (Poussot-Vassal et al., 2006), MPC (Canale et al., 2006) and LPV approaches (Poussot-Vassal et al., 2008) can be mentioned.

A different semi-active suspensions technology is based on the magnetorheological (MR) effects of certain fluids
(Wereley, 2013), usually oil. When subjected to a magnetic field, the oil exhibits an increased viscosity, to the point of becoming a viscoelastic solid: such an effect is employed to change the equivalent damping force exerted by the suspension, without the need of changing its geometry. The control input in this case is once again nonlinear and corresponds to the yield stress of the fluid, accurately adjusted by varying the magnetic field intensity with a simple electromagnet. The peculiarity of the magnetorheological technology lies in the highly non-linear characteristic of its damping force. Typically, it appears that the non-linear characteristic resembles the overall effect of a constant viscous term, plus a saturation-like term, whose amplitude is proportional to the control input.

Even in the passive configuration (namely when the control input is held constant), the magnetorheological suspension provides a nonlinear behaviour: as a consequence, controlling these devices is an even more complicated task. There are two main streams in the scientific literature: on the one side state-of-the-art solutions developed for the EH technology have been reused in the MR case, see e.g. Yao et al. (2002); Corno et al. (2019). On the other side, researchers also developed custom control solutions explicitly addressing the nonlinearity of the MR technology, see e.g. Du et al. (2005); Turnip et al. (2008); Zapateiro et al. (2011).

In this paper we set in the latter mainstream, by proposing a model-based controller design for a semi-active MR suspension, to improve the passenger comfort. With respect to the available literature, the MR suspension is here 
modelled by exploiting the saturation nonlinear elements. As a result, the control problem is addressed using the Linear Matrix Inequalities (LMIs) framework stemming from regional sector properties of the saturation function, as summarized in Tarbouriech et al. (2011). The proposed control strategy is preliminarily validated against a passive configuration and a Skyhook strategy, a consolidated switching approach for the control of EH semi-active suspensions.

The reminder of the paper is as follows: in Section 2 the vehicle and the MR suspension model are introduced, along with the road generation and the performance cost function. The benchmark strategies are presented in Section 3 and the proposed LMI-based solution is given in Section 4 . The simulations in Section 5 show the effectiveness of the proposed controller and its characteristics. Concluding remarks are finally given in Section 6 .

\section{SYSTEM MODELLING AND PERFORMANCE METRIC}

\subsection{Quarter-car model}

In order to test the proposed control strategy and compare it with known state-of-the-art solutions, a quarter car model is used, as typically proposed in the consolidated scientific literature (see e.g. Savaresi et al. (2010)).

The quarter car model parameters are tuned to match those of an SUV-like vehicle and are listed in Table 1. Indeed such vehicles benefit from the use of MR suspensions for the vertical dynamics control, given the increased ground clearance for the off-road usage, and the significant forces required at low speed to damp the high sprung mass.

The differential equations that describe the quarter car model follow:

$$
\left\{\begin{array}{l}
m_{s} \ddot{z}_{s}=-k \tilde{z}-f_{d}(\tilde{z}) \\
m_{u} \ddot{z}_{u}=-k_{t}\left(z_{u}-z_{r}\right)+k \tilde{z}+f_{d}(\dot{\tilde{z}}),
\end{array}\right.
$$

where $\tilde{z}:=z_{s}-z_{u}$. In equations (1), the suspension is modelled as a linear spring, coupled with the damper whose force depends only on the suspension stroke speed $\dot{\tilde{z}}$, as further elaborated in the following.

\begin{tabular}{||c||c|c||}
\hline Parameter & Symbol & Value \\
\hline Unsprung Mass & $m_{u}$ & $70 \mathrm{Kg}$ \\
\hline Sprung Mass & $m_{s}$ & $450 \mathrm{Kg}$ \\
\hline Suspension stiffness & $k$ & $27000 \frac{\mathrm{N}}{m}$ \\
\hline Tire stiffness & $k_{t}$ & $300000 \frac{\mathrm{N}}{\mathrm{m}}$ \\
\hline Minimum damping & $c_{\min }$ & $800 \frac{\mathrm{Ns}}{\mathrm{m}}$ \\
\hline Saturation slope & $k_{0}$ & $38000 \frac{\mathrm{Ns}}{\mathrm{m}}$ \\
\hline Maximum saturation level & $\tilde{f}_{\max }$ & $3000 \mathrm{~N}$ \\
\hline
\end{tabular}

Table 1. Quarter car and MR suspension model parameters.

\subsection{MR semi-active suspension model}

Modelling a semi-active magnetorheological suspension is a known topic in the scientific literature. There are two distinguishing features of such devices:
(1) A highly nonlinear force-speed relationship, made up by a pre-yield and a post-yield region: in the former the force increases drastically with the stroke speed whereas in the second one the force exerted by the device bends. Thanks to the semi-active capability, the pre-yield region can be increased or decreased.

(2) A hysteretic behaviour highly dependent on the fluid properties, that can be more or less pronounced and which is usually neglected when dealing with control oriented models, see for example Du et al. (2005); Do et al. (2012); Turnip et al. (2008); Zapateiro et al. (2011); Pepe et al. (2019).

In the present work, the MR damper is modelled by focusing on the nonlinear force-speed relationship, neglecting the possible hysteretic behaviour of the device. This choice is consistent with the classical scientific literature approach, as discussed, and with the objective of this paper, namely highlighting the benefits of designing a force-speed shape aware control algorithm, as compared to the state-of-the-art solutions that are typically agnostic with respect to the device characteristics.

The upper plot of Figure 1 shows an example of the typical force-speed curves of a magnetorheological suspension, for different values of the applied current. It can be seen that when the pre-yield region is null, the suspension characteristic resembles a linear one (red dash-dotted line); for higher current values, the pre-yield region expands with a significant increase of the damping force.

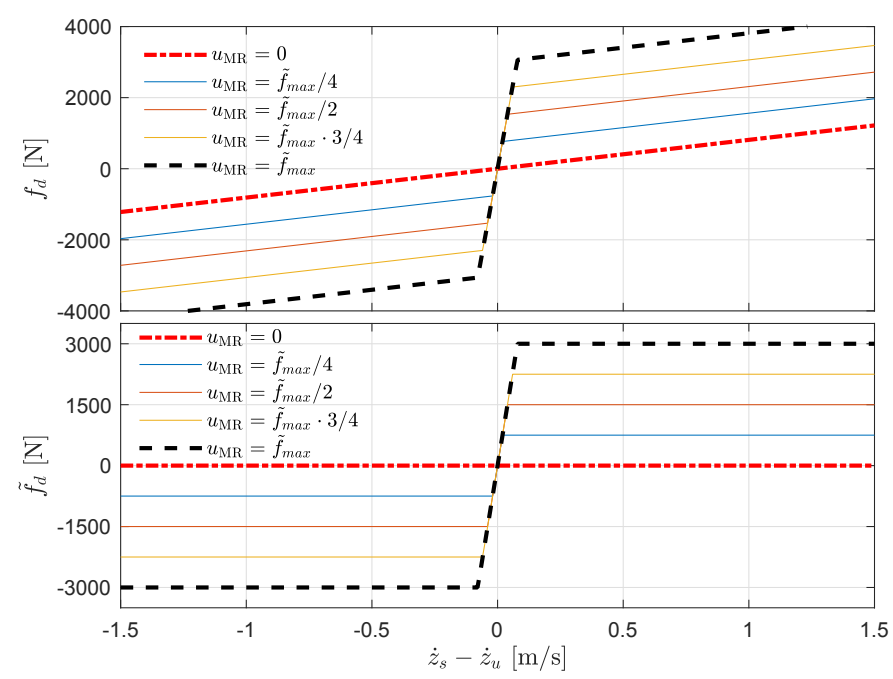

Fig. 1. Typical force-speed curve associated to a MR suspension (upper plot) and the corresponding nonlinearity (lower plot).

The mathematical model used in this work to describe the force-speed nonlinearity $f_{d}(\dot{\tilde{z}})$ of the MR damper is

$$
f_{d}=c_{\min }\left(\dot{z}_{s}-\dot{z}_{u}\right)+\tilde{f}_{d}\left(u_{\mathrm{MR}}, \dot{z}_{s}-\dot{z}_{u}\right) \text {. }
$$

which is composed by two elements: a linear one, representing the minimum damping (the red dashed-dotted line in the upper plot of Figure 1) and the nonlinear term $\tilde{f}_{d}$ accounting for the nonlinearity of the suspension. In particular, the nonlinear term can be described by the saturation-like function shown in the bottom plot of Figure 1, modelled as: 
$\tilde{f}_{d}\left(u_{\mathrm{MR}}, \dot{z}_{s}-\dot{z}_{u}\right)=\operatorname{sat}_{u_{\mathrm{MR}}}\left(k_{0}\left(\dot{z}_{s}-\dot{z}_{u}\right)\right), \quad u_{\mathrm{MR}} \in\left[0, \tilde{f}_{\mathrm{max}}\right]$.

where $\operatorname{sat}_{\bar{u}}(s):=\max \{\min \{s, \bar{u}\},-\bar{u}\}$ represents the symmetric scalar saturation function having limits $\pm \bar{u}$, and the constant $k_{0}$ corresponds to the slope of the saturation function near the origin. Notice that in equation (3) the control variable of the semi-active suspension has been explicitly defined, and corresponds to the saturation level $u_{\mathrm{MR}}$.

The parameters that completely define equations (2) and (3) are listed in Table 1: they have been chosen according to the results presented in Goldasz and Dzierżek (2016), to represent an average automotive MR damper, that suites also for an SUV vehicle.

\subsection{Performance characterization and road definition}

The performances of the passive suspension and the semiactive control strategies are compared in terms of passenger perceived comfort. As widely accepted in the scientific literature, a means to quantitatively evaluate this aspect is the index $J$ corresponding to the rms of the output acceleration $y=\ddot{z}_{s}$ :

$$
J\left(\ddot{z}_{s}\right)=\left(\frac{1}{T} \int_{0}^{T}\left|\ddot{z}_{s}(\tau)\right|^{2} d \tau\right)^{\frac{1}{2}}
$$

where $T$ is the duration of the simulation (or experiment).

The driving comfort is evaluated by simulating the quarter car model (1) for a road profile $z_{r}$ generated according to the ISO-8608 standard, as presented in Agostinacchio et al. (2014). All the simulations and the results of the next sections are achieved by considering a road profile of class C-D (representing a light off-road condition, see Agostinacchio et al. (2014)), with a velocity of $v=90 \frac{\mathrm{km}}{\mathrm{h}}$. Figure 2 shows an example of the considered road profile, as a function of the longitudinal travelled distance.

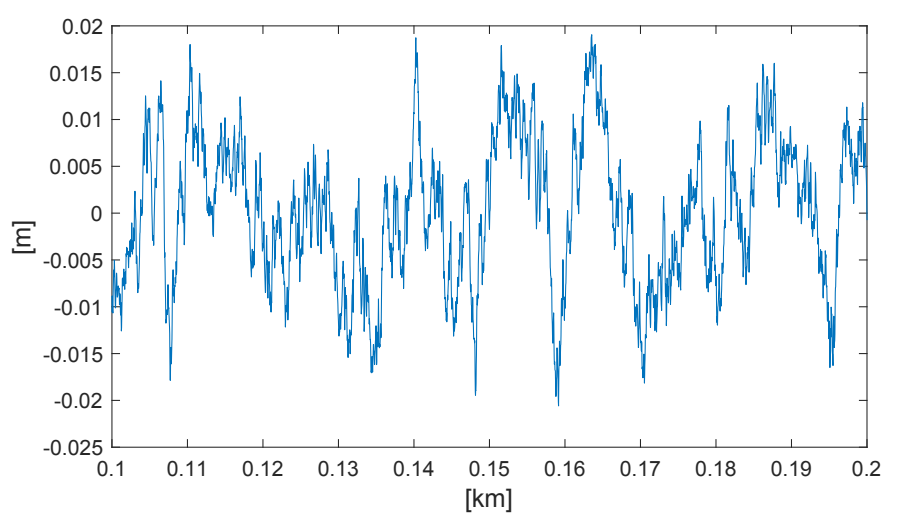

Fig. 2. Example of a generated road profile.

In order to avoid the variability due to the specific realization of the random Gaussian noise, the considered road has been selected to be sufficiently long (that is, $2.5 \mathrm{~km}$ long) so that the functional $J$ directly benefits of an averaging effect.

\subsection{Overall system model}

Combining all the elements of the simulation framework discussed above, it is possible to write a compact expression for the considered system. By defining the state variable vector $x=\left[\begin{array}{llll}z_{s} & \tilde{z} & \dot{z}_{s} & \dot{\tilde{z}}\end{array}\right]^{\top}$, considering as external input the excitation input $z_{r}$ coming from the road, and as the performance output the vertical acceleration $y=\ddot{z}_{s}$, the overall system can be described by the following statespace equations:

$$
\Sigma_{\mathrm{MR}}:\left\{\begin{array}{l}
\dot{x}=A\left(c_{\mathrm{min}}\right) x-B \tilde{f}_{d}\left(u_{\mathrm{MR}}, \dot{\tilde{z}}\right)+E z_{r} \\
y=\ddot{z}_{s}=C\left(c_{\min }\right) x-D \tilde{f}_{d}\left(u_{\mathrm{MR}}, \dot{\tilde{z}}\right)
\end{array}\right.
$$

with

$\left[\begin{array}{l|l|l}A\left(c_{\min }\right) & B & E \\ \hline C\left(c_{\min }\right) & D & \end{array}\right]=$

$\left[\begin{array}{cccc|c|c}0 & 0 & 1 & 0 & 0 & 0 \\ 0 & 0 & 0 & 1 & 0 & 0 \\ 0 & -\frac{k}{m_{s}} & 0 & -\frac{c_{\min }}{m_{s}} & \frac{1}{m_{s}} & 0 \\ \frac{k_{t}}{m_{u}}-\frac{k}{m_{s}}-\frac{k_{t}+k}{m_{u}} & 0 & -\frac{c_{\min }\left(m_{s}+m_{u}\right)}{m_{u} m_{s}} & \frac{m_{s}+m_{u}}{m_{u} m_{s}} & -\frac{k_{t}}{m_{u}} \\ \hline 0 & -\frac{k}{m_{s}} & 0 & -\frac{c_{\min }}{m_{s}} & \frac{1}{m_{s}} & \end{array}\right]$

The proposed model is generically nonlinear, due to the saturation that defines $\tilde{f}_{d}\left(u_{\mathrm{MR}}, \dot{\tilde{z}}\right)$; when $u_{\mathrm{MR}}=0$ the model turns into a linear one, with minimum suspension damping, equal to $c_{m i n}$.

\section{PASSIVE AND SEMI-ACTIVE BENCHMARKS}

The control strategy proposed in Section 4 is tested against two classical benchmarks when dealing with semi-active suspensions: the fully passive and the Skyhook semi-active control strategy.

\subsection{Passive benchmark}

The basic comparison of any semi-active control strategy is the corresponding passive suspension one. It is well-known that the choice of the suspension damping, in the passive case, undergoes a performances trade-off and the best comfort performances are found for intermediate (neither too low, nor too high) damping values. Thus, a sensitivity analysis of the comfort index with respect to different, constant, values of the control variable $u_{\mathrm{MR}}$ has been performed: as a matter of fact, the best performances are obtained for the passive case $\left(J \cong 1.6 \frac{\mathrm{m}}{\mathrm{s}^{2}}\right)$ corresponding to setting $u_{\mathrm{MR}}=0$. This result is consistent with the selected value of the linear damping $c_{\min }$ which is very close to the optimal value that can be obtained for a linear suspension; within this perspective it is clear that introducing an additional MR damping force in the system (by using values of $u_{\mathrm{MR}}>0$ ) worsens the comfort performances in the same way as it would happen for the linear case.

\subsection{Skyhook benchmark}

The Skyhook semi-active controller is a classical comfortoriented control strategy developed for semi-active suspensions, Savaresi et al. (2010). In particular, the so called two-state Skyhook is a switching control strategy that 
switches the suspension damping between its minimum and maximum values, according to the following law:

$$
c= \begin{cases}c_{\min }, & \text { if } \dot{z}_{s} \dot{\tilde{z}} \leq 0 \\ c_{\max }, & \text { if } \dot{z}_{s} \dot{\tilde{z}}>0 .\end{cases}
$$

Despite being conceived and designed for linear semi-active suspensions (typically realised with the Electro-Hydraulic technology) this control strategy has been directly applied also to the MR suspensions, as recalled in the introduction. In the present work, we consider this strategy as another benchmark to be compared with the LMI-based solutions of the next sections.

The MR Skyhook control strategy mimics the EH one, but rather than switching between two damping factors $c_{\min }$ and $c_{\max }$, the control input $u_{\mathrm{MR}}$ in (3) switches directly between 0 and $\tilde{f}_{\max }$ :

$$
u_{\mathrm{MR}}= \begin{cases}0, & \text { if } \dot{z}_{s} \dot{\tilde{z}} \leq 0 \\ \tilde{f}_{\text {max }}, & \text { if } \dot{z}_{s} \dot{\tilde{z}}>0\end{cases}
$$

When simulating the quarter-car model with the control law (7) the resulting performance index is $J \cong 2.35 \frac{\mathrm{m}}{\mathrm{s}^{2}}$, even higher than the passive benchmark. On the one side this result is consistent with the Skyhook formulation which is designed to limit the chassis velocity, rather than its accelerations. However, such a performance worsening is amplified in the MR case, mainly due to the nonlinearities of the force-speed maps of these dampers.

The obtained result motivates the development of the MR control strategy proposed in the next section, which explicitly includes the shape of the force-speed maps in the controller design.

\section{LMI-BASED CONTROLLER}

The results of the previous section show that the twostates skyhook controller merely applied to the MR suspension is not effective at improving the passive performance. Motivated by this fact, in this section we design a specific controller for the MR suspension by exploiting Linear Matrix Inequality (LMI) techniques certifying stability and performance of systems subject to saturation nonlinearities Tarbouriech et al. (2011). Since those techniques mostly focus on designing a saturated state feedback $K$ in dynamical systems of type $\dot{x}=A x+B \operatorname{sat}_{\bar{u}}(K x)$, for a fixed value of $\bar{u}$, we perform below some manipulations of the MR dynamics (1), (2) to partially match this standard structure.

\subsection{Approximate representation of $\tilde{f}_{d}$}

To the end of providing a representation compatible with existing LMI-based techniques for feedback control design, we consider the MR suspension output in (3), which enters linearly in the dynamics $(5)$ (recall that $c_{\min }$ is constant) and we provide the following approximate representation

$$
\tilde{f}_{d}=v_{\mathrm{MR}}+\operatorname{sat}_{\frac{\tilde{f}_{\max }}{2}}\left(k_{0} \dot{\tilde{z}}\right), v_{\mathrm{MR}} \in\left[-\frac{\tilde{f}_{\max }}{2}, \frac{\tilde{f}_{\max }}{2}\right] \text {, }
$$

where $v_{\mathrm{MR}}=\left(u_{\mathrm{MR}}-\frac{f_{\max }}{2}\right) \operatorname{sgn}(\dot{\tilde{z}})$ can be freely assigned in the interval $\left[-\frac{\tilde{f}_{\max }}{2}, \frac{\tilde{f}_{\text {max }}}{2}\right]$ by suitably assigning

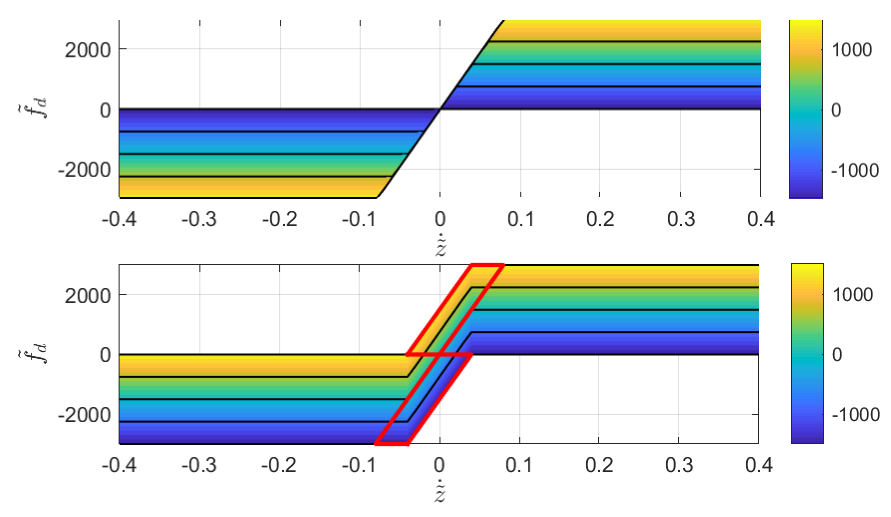

Fig. 3. Variable saturation model (upper plot) and double saturation model (lower plot) of the MR suspension. The red areas in the lower graph are regions where the double saturation model is not accurate.

$u_{\mathrm{MR}} \in\left[0, f_{\max }\right]$ in $(3)$. With model (8) it becomes then convenient to design the bounded equivalent input $v_{\mathrm{MR}}$ as sat $\frac{\tilde{f}_{\text {max }}}{2}\left(K_{2} x\right)$, where $K_{2}$ is a four element row vector representing a state feedback gain to be optimized.

In particular, as shown in Figure 3 , the force $\tilde{f}_{d}$ generated by the actual suspension (3) driven by the input

$$
\begin{aligned}
u_{\mathrm{MR}} & =\frac{\tilde{f}_{\text {max }}}{2}+\operatorname{sgn}(\dot{\tilde{z}}) \operatorname{sat}_{\frac{\tilde{f}_{\max }}{2}}\left(\left[k_{1} k_{2} k_{3} k_{4}\right] x\right), \\
& =\frac{\tilde{f}_{\max }}{2}+\operatorname{sgn}(\dot{\tilde{z}}) \underbrace{\operatorname{sat}_{\frac{\tilde{f}_{\max }}{2}}\left(K_{2} x\right)}_{v_{\mathrm{MR}}},
\end{aligned}
$$

perfectly coincides with the force exerted by the double saturation model (8) when $\left|v_{\mathrm{MR}}\right| \geq \frac{\tilde{f}_{\max }}{2}$, namely whenever $\left|K_{2} x\right| \geq \frac{\tilde{f}_{\max }}{2}$. It turns out that the important feature ensured by the actual model (3) as compared to the approximated one (8) is to remove points from the graph of Figure 3 corresponding to injection of energy (namely the points in the second and fourth quadrant) so that the predicted performance $J$ by the approximate model is not very different from the one of the actual model (this emerges from our numerical simulations).

\subsection{LMI-based Controller design}

Based on the approximated MR model (8) derived in the previous section and on the saturated state-feedback selection for $v_{\mathrm{MR}}$ given in (9), we may write system (5), (8) as:

$$
\begin{aligned}
& \dot{x}=A\left(c_{\text {min }}\right) x-B \operatorname{sat}_{\frac{\tilde{f}_{\text {max }}}{2}}\left(K_{1} x\right)-B \operatorname{sat}_{\frac{\tilde{f}_{\text {max }}}{2}}\left(K_{2} x\right)+E z_{r} \\
& z=C\left(c_{\min }\right) x-D \operatorname{sat}_{\frac{\tilde{f}_{\text {max }}}{2}}\left(K_{1} x\right)-D \operatorname{sat}_{\frac{\tilde{f} \max }{2}}\left(K_{2} x\right),
\end{aligned}
$$

where matrix $K_{1}$ is fixed and provides the second contribution in (8), while matrix $K_{2}$ is to be designed and corresponds to the selection of $v_{\mathrm{MR}}$ in (8). In particular,

$$
\left[\begin{array}{l}
K_{1} \\
K_{2}
\end{array}\right]=\left[\begin{array}{cccc}
0 & 0 & 0 & k_{0} \\
\hline k_{1} & k_{2} & k_{3} & k_{4}
\end{array}\right],
$$

where we may exploit the degree of freedom of designing its second row $K_{2}$. In the rest of this section, to simplify the notation, we will write $A$ (respectively, $C$ ) in place of $A\left(c_{\min }\right)$ (respectively $\left.C\left(c_{\min }\right)\right)$, and sat in place of sat $\frac{\tilde{f}_{\max }}{2}$ 
because all the saturation limits correspond to the same value $\frac{\tilde{f}_{\max }}{2}$.

We provide below two control design techniques, ensuring local asymptotic stability properties and suitable optimized disturbance rejection properties measured in terms of $\mathcal{L}_{2}$ and $\mathcal{H}_{2}$ performance.

When focusing on regional $\mathcal{L}_{2}$ performance, we solve the following optimization problem, for a fixed value of $s$, which allows adjusting the size $\left\|z_{r}\right\|_{2}$ of the maximum disturbance taken into account in the regional $\mathcal{L}_{2}$ gain characterization.

$$
\begin{gathered}
\gamma_{L}^{2}(s):=\min _{Q=Q^{\top}>0, X, U_{1}, U_{2}, Y_{1}, Y_{2}, \gamma} \gamma^{2} \text { subject to: } \\
\operatorname{He}\left[\begin{array}{ccccc}
\left(A-B K_{1}\right) Q-B X & B U_{1} & B U_{2} & E & 0 \\
K_{1} Q+Y_{1} & -U_{1} & 0 & 0 & 0 \\
X+Y_{2} & 0 & -U_{2} & 0 & 0 \\
0 & 0 & 0 & -\frac{I}{2} & 0 \\
\left(C-D K_{1}\right) Q-D X & D U_{1} & D U_{2} & 0 & -\gamma^{2} \frac{I}{2}
\end{array}\right]<0, \\
{\left[\begin{array}{cc}
Q & Y_{1}^{\top} \\
Y_{1} & \tilde{f}_{\max }^{2} /\left(4 s^{2}\right)
\end{array}\right] \geq 0, \quad\left[\begin{array}{ccc}
Q & Y_{2}^{\top} \\
Y_{2} & \tilde{f}_{\max }^{2} /\left(4 s^{2}\right)
\end{array}\right] \geq 0}
\end{gathered}
$$

The following proposition establishes desirable properties when implementing $K_{2}=X Q^{-1}$ in (10), stemming from a solution to the optimization (11).

Proposition 1. Given a scalar $s>0$, consider any solution to the optimization (11). Then selecting $K_{2}=X Q^{-1}$, the closed loop (10) is such that for any input $z_{r}$ having $\mathcal{L}_{2}$ norm $\left\|z_{r}\right\|_{2} \leq s$, it holds that the performance output satisfies $\|z\|_{2} \leq \gamma_{L}(s)\left\|z_{r}\right\|_{2}$.

The second LMI-based design that we propose for the gain $K_{2}$ is based on a performance index of the $\mathcal{H}_{2}$ type (see, e.g., (Boyd et al., 1994, §10.1.1 and page 137)), which is motivated by the fact that there is no feedthrough term between $z_{r}$ and $z$. In particular, we propose below to minimize the $\mathcal{H}_{2}$ norm of the linear transfer function seen by the disturbance $z_{r}$ when the amplitude is small enough to not activate the deadzone functions. This norm can be minimized while, at the same time, ensuring asymptotic stability of the origin for the saturated closed loop, by solving the following optimization

$$
\begin{aligned}
\gamma_{H}^{2}(s):= & \min _{Q=Q^{\top}>0, X, U_{1}, U_{2}, Y_{1}, Y_{2}, \gamma} \gamma^{2} \text { subject to: } \\
& \operatorname{He}\left[\begin{array}{ccc}
\left(A-B K_{1}\right) Q-B X & B U_{1} & B U_{2} \\
K_{1} Q+Y_{1} & -U_{1} & 0 \\
X+Y_{2} & 0 & -U_{2}
\end{array}\right]<0 \\
& \operatorname{He}\left[\begin{array}{cc}
\left(A-B K_{1}\right) Q-B X & 0 \\
\left(C-D K_{1}\right) Q-D X & -\frac{1}{2}
\end{array}\right]<0 \\
& {\left[\begin{array}{ll}
\gamma^{2} & E^{\top} \\
E & Q
\end{array}\right]>0, } \\
& {\left[\begin{array}{ll}
Q & Y_{1}^{\top} \\
Y_{1} & \tilde{f}_{\max }^{2} /\left(4 s^{2}\right)
\end{array}\right] \geq 0, \quad\left[\begin{array}{cc}
Q & Y_{2}^{\top} \\
Y_{2} & \tilde{f}_{\max }^{2} /\left(4 s^{2}\right)
\end{array}\right] \geq 0 }
\end{aligned}
$$

where the optimized variable $\gamma$ is an upper bound on the maximum energy experienced at the output $\|z\|_{2}$ as compared to the size of the initial condition $x_{0}=\eta E$, selected in the subspace spanned by the input matrix $E$, namely $\|z\|_{2} \leq \gamma|\eta|$. In optimization (12), the positive parameter $s$ establishes an indirect bound on the size of the ellipsoidal set of initial conditions that is guaranteed to be contained in the basin of attraction of the origin.

The following proposition establishes desirable properties when implementing $K_{2}=X Q^{-1}$ in (10), stemming from a solution to the optimization (12).

Proposition 2. Given a scalar $s>0$, consider any solution to the optimization (12). Then selecting $K_{2}=X Q^{-1}$, the closed loop (10) is (locally) asymptotically stable to the origin with the set $x^{\top} Q^{-1} x \leq s^{2}$ being contained in the basin of attraction. Moreover, any (small signal) response remaining below the saturation limits for all times and starting in $x_{0}=\eta E$ for some $\eta \in \mathbb{R}$, satisfies $\|z\|_{2} \leq \gamma_{H}(s)|\eta|$

The proofs of Propositions 1 and 2, are based on the use of the regional sector conditions discussed in Tarbouriech et al. (2011) and are here omitted for space constraints.

\section{SIMULATION RESULTS WITH LMI-BASED CONTROLLERS}

Using the numerical parameters in Table 1, both the LMI optimization problems in (11) and (12) illustrated in Section 4 have been solved in Matlab, using the solver MOSEK over a range of possible values for the variable $s$ between 0.1 and 1.5, which provides interesting trends for the quantity $\gamma$.

For each one of these solutions (corresponding to a specific selection of the four gains in $K_{2}$ ), the performance criterion $J$ in (4) has been evaluated by simulation, by implementing two scenarios, corresponding to the regions represented in Figure 3:

- Double Saturation (DS) Model: represented by the bottom curve of Figure 3, corresponds to directly implementing the closed loop (10), namely the closed loop for which Propositions 1 and 2 provide stability and performance guarantees;

- Variable Saturation (VS) Model: more faithful to reality, corresponds to only allowing pairs $\left(\tilde{f}_{d}, \dot{\tilde{z}}\right)$ to take values in the actual region spanned by the feasible input-output pairs of the real suspension. This set of simulations has been performed by running model (5) with input $\tilde{f}_{d}$ obtained from (3) when the suspension input $u_{\mathrm{MR}}$ is selected as in (9).

Figure 4 reports the curves obtained from the evaluation of $J$ in the various cases. A first result stemming from this figure is that the $\mathcal{L}_{2}$-based optimization does not provide interesting results because it gives large values of $J$. The $\mathcal{H}_{2}$-based scheme, instead, provides a minimum $\operatorname{cost} J^{*}=1.48 \frac{\mathrm{m}}{\mathrm{s}^{2}}$ obtained for $s^{*}=0.1$, which is desirably lower than the minimum cost achieved with the Skyhook linear controller (around $2.35 \frac{\mathrm{m}}{\mathrm{s}^{2}}$ ) and the passive solution $\left(1.6 \frac{m}{s^{2}}\right)$, leading to an overall comfort performance increase of $\approx 7 \%$. The gain $K_{2}$ resulting from this optimal point corresponds to

$$
K_{2}=\left[\begin{array}{llll}
-18901-4592022704-36338
\end{array}\right] \text {. }
$$

A second interesting result that can be observed from Figure 4 is that the green-black and red-blue curve pairs are very close to each other, thereby showing that the DS and VS saturation models considered in our validation 


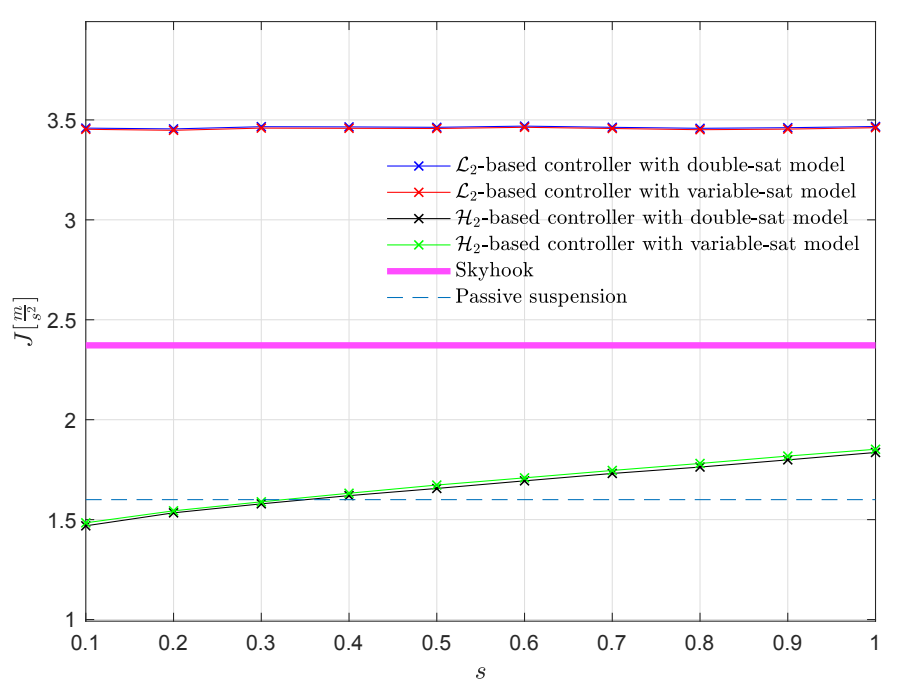

Fig. 4. Evolution of the index $J$ as a function of $s$ for the $\mathcal{L}_{2^{-}}$and the $\mathcal{H}_{2}$-based syntheses evaluated with the VS and the DS models. The horizontal purple line is the value of $J$ obtained from the skyhook method of Section 3.

provide essentially equivalent results, despite the presence of infeasible areas (those circled in red in Figure 3) when using the DS model. Such an evidence confirms that the approximation in Section 4.1 is reasonable and the controller design is effective even with this approximation.

To conclude, the simulation tests confirm that the proposed LMI-based techniques stemming from the approximated model of Section 4 are effective in providing a useful gain tuning technique for the semi-active MR suspension.

\section{CONCLUSIONS}

In this paper we addressed the control synthesis for a semiactive suspension designed based on magnetorheological effects. We first provided a performance characterization based on a prototype simulation scenario, then showed that the classical Skyhook control technique does not provide significant improvements as compared to the passive scenario (when the suspension input is kept constant), and we finally presented an approximated model allowing for optimized tuning of a state feedback gain, based on a semidefinite program written in terms of Linear Matrix Inequalities for efficient tuning. Simulation results confirmed the effectiveness of the proposed technique.

\section{REFERENCES}

Agostinacchio, M., Ciampa, D., and Olita, S. (2014). The vibrations induced by surface irregularities in road pavements - a matlabß approach. European Transport Research Review, 6(3), 267-275.

Boyd, S., El Ghaoui, L., Feron, E., and Balakrishnan, V. (1994). Linear matrix inequalities in system and control theory, volume 15. Siam.

Canale, M., Milanese, M., and Novara, C. (2006). Semiactive suspension control using "fast" model-predictive techniques. IEEE Transactions on control systems technology, 14(6), 1034-1046.

Corno, M., Galluppi, O., Panzani, G., Sinigaglia, A., Capuano, P., Cecconi, J., and Savaresi, S.M. (2019). Design and validation of a full body control semi-active suspension strategy for a supercar. IFAC-PapersOnLine, 52(5), 667-672.

Do, A.L., Sename, O., and Dugard, L. (2012). Lpv modeling and control of semi-active dampers in automotive systems. In Control of linear parameter varying systems with applications, 381-411. Springer.

Du, H., Sze, K.Y., and Lam, J. (2005). Semi-active h $\infty$ control of vehicle suspension with magneto-rheological dampers. Journal of Sound and Vibration, 283(3-5), 981-996.

Goldasz, J. and Dzierżek, S. (2016). Parametric study on the performance of automotive mr shock absorbers. IOP Conference Series: Materials Science and Engineering, 148.

Pepe, G., Roveri, N., and Carcaterra, A. (2019). Experimenting sensors network for innovative optimal control of car suspensions. Sensors, 19(14), 3062.

Poussot-Vassal, C., Sename, O., Dugard, L., Gaspar, P., Szabo, Z., and Bokor, J. (2008). A new semi-active suspension control strategy through lpv technique. Control Engineering Practice, 16(12), 1519-1534.

Poussot-Vassal, C., Sename, O., Dugard, L., RamirezMendoza, R., and Flores, L. (2006). Optimal skyhook control for semi-active suspensions. IFAC Proceedings Volumes, 39(16), 608-613.

Savaresi, S.M. and Spelta, C. (2007). Mixed sky-hook and add: Approaching the filtering limits of a semi-active suspension. Journal of dynamic systems, measurement, and control, 129(4), 382-392.

Savaresi, S., Poussot-Vassal, C., Spelta, C., Sename, O., and Dugard, L. (2010). Semi-active suspension control design for vehicles. Elsevier.

Tarbouriech, S., Garcia, G., Gomes da Silva Jr, J., and Queinnec, I. (2011). Stability and stabilization of linear systems with saturating actuators. Springer Science \& Business Media.

Turnip, A., Hong, K.S., and Park, S. (2008). Control of a semi-active mr-damper suspension system: A new polynomial model. IFAC Proceedings Volumes, 41(2), 4683-4688.

Wereley, N. (2013). Magnetorheology: advances and applications, volume 6. Royal Society of Chemistry.

Yao, G., Yap, F., Chen, G., Li, W., and Yeo, S. (2002). $\mathrm{Mr}$ damper and its application for semi-active control of vehicle suspension system. Mechatronics, 12(7), 963973.

Zapateiro, M., Pozo, F., Karimi, H.R., and Luo, N. (2011). Semiactive control methodologies for suspension control with magnetorheological dampers. IEEE/ASME Transactions on mechatronics, 17(2), 370-380. 